Research Paper

\title{
Twistl Enhances Hypoxia Induced Radioresistance in Cervical Cancer Cells by Promoting Nuclear EGFR Localization
}

\author{
Hua Xiong ${ }^{*}$, Xin $\mathrm{Nie}^{1 *}$, Yanmei Zou ${ }^{1}$, Chen Gong ${ }^{1}$, Yang $\mathrm{Li}^{1}$, Hua Wu ${ }^{1}$, Hong Qiu ${ }^{1}$, Lin Yang1, Liang \\ Zhuang1, Peng Zhang'1, Jing Zhang1, Yihua Wang², Huihua Xiong1凶 \\ 1. Department of Oncology, Tongji Hospital, Tongji Medical College, Huazhong University of Science and Technology, Wuhan, Hubei Province, China. \\ 2. Biological Sciences, Faculty of Natural and Environmental Sciences, University of Southampton, Southampton SO17 1BJ, UK. \\ *These authors contribute to this paper equally. \\ $\triangle$ Corresponding author: Tongji Hospital, Tongji Medical College, Huazhong University of Science and Technology, Wuhan 430030, Hubei province, China. \\ E-mail: xionghuihua@medmail.com.cn. \\ (c) Ivyspring International Publisher. This is an open access article distributed under the terms of the Creative Commons Attribution (CC BY-NC) license \\ (https://creativecommons.org/licenses/by-nc/4.0/). See http://ivyspring.com/terms for full terms and conditions.
}

Received: 2016.06.26; Accepted: 2016.10.29; Published: 2017.02.09

\begin{abstract}
Twistl is a crucial transcription factor that regulates epithelial mesenchymal transition and involves in metastasis. Recent evidence suggests that Twistl plays important role in hypoxia-induced radioresistance, but the underlying mechanism remains elusive. Here we investigated the change of Twistl expression in human cervical squamous cancer cell line $\mathrm{SiH}$ a after hypoxia treatment. We also explored the role of Twistl in radioresistance by manipulating the expression level of Twist 1 . We observed that hypoxia treatment elevated the expression of Twistl in SiHa cells. Knockdown of Twistl with siRNA increased the radiosensitivity of $\mathrm{SiHa}$ cells under hypoxia condition, accompanied by reduced levels of nuclear Epidermal Growth Factor Receptor (EGFR) and DNA-dependent protein kinase (DNA-PK). Conversely, overexpression of Twistl led to increased radioresistance of $\mathrm{SiHa}$ cells, which in turn increased nuclear EGFR localization and expression levels of nuclear DNA-PK. Moreover, concomitant high expression of hypoxia-inducible factor-1 $\alpha$ (HIF-1 $\alpha$ ) and Twistl in primary tumors of cervical cancer patients correlated with the worse prognosis after irradiation treatment. Taken together, these data provide new insights into molecular mechanism underlying hypoxia-induced radioresistance in cervical cancer cells, and suggest that Twistl is a promising molecular target to improve the efficacy of cancer radiotherapy.
\end{abstract}

Key words: Twist1; Hypoxia; EGFR; radioresistance.

\section{Introduction}

Cervical cancer remains to be the fourth most common type of cancer and presents the fourth leading cause of cancer death in women globally. It is estimated that 528,000 new cases of cervical cancer occur and 266,000 deaths are caused by cervical cancer annually around the world [1]. Radiotherapy has been widely applied in women with locally advanced cervical cancer [2]. However, cancer radiosensitivity is determined not only by intrinsic sensitivity of cancer cells but also by tumor microenvironment. Hypoxia, a condition of low oxygen level, has been recognized as a characteristic feature of solid tumors. About 50-60\% of locally advanced solid tumors, including cervical cancer, comprise hypoxic region which is often associated with resistance to radiotherapy and poor survival [3-5].

Hypoxia-inducible factor 1 (HIF-1) consists of a and $\beta$ subunits and functions as an essential regulator of molecular response to hypoxia. HIF-1a is induced immediately after a decrease in oxygen tension and 
enables tumor cells to endure a hypoxic microenvironment. Accumulating evidences have shown that the overexpression of HIF-1a is responsible for radio-resistance among different types of carcinoma and cancer cell lines [3, 6]. Moreover, the inhibition of HIF-1a expression by short interfering RNA (siRNA) could reverse hypoxia mediated resistance to radiotherapy by cancer cells [7]. However, the molecular mechanisms underlying hypoxia-induced radiation resistance remain elusive.

Twist1 is a basic-helix-loop-helix transcription factor and acts as a key regulator of epithelial-mesenchymal transition (EMT), which promotes tumor invasion and metastasis [8]. Inhibiting the expression of Twist1 could reverse hypoxia-mediated EMT effectively [9, 10]. Furthermore, siRNA mediated Twist1 knockdown enhanced ionizing radiation (IR) induced cell death in non-small-cell lung cancer (NSCLC) cells [11]. These studies imply that Twist1 plays important roles in hypoxia-induced EMT and metastasis. Knockdown of Twist1 may ameliorate hypoxia induced radioresistance. Recent evidence suggests that nuclear EGFR was highly associated with the resistance to radiation $[12,13]$. After the exposure to irradiation, EGFR is translocated into the nucleus and forms a complex with DNA-dependent protein kinase DNA-PK [14], which is composed of catalytic subunit (DNA-PKcs) and $\mathrm{Ku}$ heterodimer (Ku70 and Ku80) [15]. In this study, we aimed to investigate the role of Twist1 in the regulation of hypoxia induced radiosensitivity of cervical cancer, and explored the underlying mechanisms. By using human cervical carcinoma cell line $\mathrm{SiHa}$, we demonstrated that Twist1 knockdown could sensitize hypoxic SiHa cells to IR through decreasing the nuclear localization of EGFR and inhibiting DNA-PKcs activation. Moreover, Twist1 overexpression in cervical cancer patients correlated with the worse prognosis after irradiation treatment. Our data suggests that Twist 1 is a promising molecular target to improve the efficacy of cancer radiotherapy.

\section{Materials and Methods}

\section{Cell culture}

Human cervical carcinoma cell line $\mathrm{SiHa}$, and 293T/17 cells were purchased from the American Type Culture Collection (ATCC), and cultured in DMEM (Hyclone, Thermo scientific, USA) supplemented with $10 \%$ fetal bovine serum (Gibco, Life Technologies, USA) and $1 \%$ penicillin-streptomycin solution (Hyclone, Thermo scientific, USA) at $37^{\circ} \mathrm{C}$ in a $5 \% \mathrm{CO}_{2}$ incubator. For hypoxia treatment, cells were incubated in a humidified $\mathrm{O}_{2}$ control incubator $\left(5 \% \mathrm{CO}_{2}, 1 \% \mathrm{O}_{2}\right)$ for indicated time or cultured with cobalt chloride $\left(\mathrm{CoCl}_{2}\right.$, Sigma) solution as reported previously [16].

\section{Colony formation assay}

Cells were counted and seeded at colony density in 6-well plates. After incubation with in a humidified $\mathrm{O}_{2}$ control incubator $\left(1 \% \mathrm{O}_{2}\right.$ or $\left.19 \% \mathrm{O}_{2}\right)$ for $8 \mathrm{~h}$, cells were irradiated with different doses $(0-8 \mathrm{~Gy})$ at $25^{\circ} \mathrm{C} .4$ $\mathrm{h}$ after IR, the medium was changed and the cells were cultured for 10 to 14 days to get viable colonies. Cells were stained with $0.1 \%$ crystal violet (SCR, Shanghai, China) for 15 min and counted.

\section{Vector construction and lentiviruses infection}

The sequences of Twist1-targeting siRNA were as follows: siRNA1: $5^{\prime}$-CCT TCT CGGTCT GGA GGA T-3'; siRNA2: 5'-TGG ACT CCA AGA TGG CAA G-3'; siRNA3:5'-CGC AGT CTT ACG AGG AGC T-3'; and the sequences of mock control siRNA were: 5'-TTC TCC GAA CGT GTC ACG T-3'. Human Twist1 cDNA clone was purchased from GeneTechnology and was amplified by RT-PCR. The pGCL-EGFP (enhanced green fluorescent protein)/pHelper1.0/pHelper2.0 were purchased from GeneTechnology (Shanghai, China). The siRNA sequences and Twist1 cDNA were subcloned into pGCL-EGFP vector.

Lentiviruses were prepared by transfecting the plasmids into $293 \mathrm{~T}$ cells using Lipofectamine 2000 (Invitrogen, CA, USA) to obtain LV-Twist1-siRNA-1, LV-Twist1-siRNA-2, LV-Twist1-siRNA-3, LV-Twist1+ and LV-Vector. Finally, lentiviruses were harvested from the supernatants of infected 293T cells, and then the virus titer was determinated. Next, the lentiviruses were added to $\mathrm{SiHa}$ cells with polybrene (final concentration: $50 \mu \mathrm{g} / \mathrm{ml}$, Sigma). The efficiency of transduction was observed under a fluorescence microscope $72 \mathrm{~h}$ after infection.

\section{Western blot analysis}

Cells were harvested with ice-cold PBS and then lysed in RIPA lysis buffer (Beyotime, China) for 40 min on ice. Nuclear extracts were extracted using the commercial kit (NE-PER, Thermo, USA) according to the manufacturer's instructions. The protein lysates were separated by SDS-PAGE and electroblotted onto PVDF membranes (Immobilon). After blocking with $10 \%$ fat-free milk at room temperature for $1 \mathrm{~h}$, the membranes were incubated with primary antibodies mouse anti-Twist1 (1:50, ab50887, Abcam), rabbit anti-gamma $\operatorname{H2AX}(1: 1000$, ab 11174, Abcam), rabbit anti- H2AX (1:1000, ab 11175, Abcam), mouse anti-HIF-1a (1:250, 610959, BD), rabbit anti-EGFR (1:1000, \#4267, Cell Signaling Technology), mouse anti-DNA-PKcs (1:1000, sc-390495, Santa Cruz), 
mouse anti-Lamin B1 (1:500, 66095-1, Proteintech), mouse anti-GAPDH (1:1000, KM9002, Tianjin Sungene Biotech) or mouse anti- $\beta$-actin (1:1000, KM9001, Tianjin Sungene Biotech), and corresponding secondary antibodies (Biosharp). Finally, the blots were detected by Super-Signal West Femto kit (34095, Thermo) and analyzed by Image Lab (Bio-Rad).

\section{Confocal microscopy}

SiHa/Twist1-siRNA, SiHa/Twist1+ and negative control cells were plated onto glass coverslips. After $24 \mathrm{~h}$ cell culture, the cells were irradiated with single dose 5 Gy after 8-h preincubation under hypoxia or normoxia. The coverslips were fixed in $4 \%$ paraformaldehyde for 10 min, permeabilized with $0.1 \%$ Triton X-100 for $15 \mathrm{~min}$ and blocked with $0.5 \%$ BSA for $1 \mathrm{~h}$ at room temperature. Next, the coverslips were incubated with primary antibodies against Twist1 (1:50, ab50887, Abcam) or EGFR (1:50, \#4267, Cell Signaling Technology) at $4^{\circ} \mathrm{C}$ overnight and then washed and incubated with Alexa Fluor 568 (1:50, Goat anti-mouse) or Alexa Fluor 647 (1:50, Goat anti-rabbit). Finally, cell nuclei were stained with $4^{\prime}$, 6-diamidino-2-phenylindole (DAPI, Boster). The coverslips were examined under confocal laser scanning microscope.

\section{Immunohistochemistry analysis}

The immunostaining assay was conducted on tumor samples which were fixed in $10 \%$ neutral formalin solution immediately after dissection and embedded in paraffin. Then sections were cut into fragments of $6 \mathrm{um}$, deparaffinized and hydrated. Tissues on slides were permeabilized by $0.5 \%$ Triton X-100 and incubated overnight at $4^{\circ} \mathrm{C}$ with primary antibodies Twist 1 and HIF-1a, respectively. The slides were washed by PBS for 5 times and incubated with 1:200 dilution of secondary biotinylated antibody for 2 hours at room temperature. All slides were developed using horseradish peroxidase-streptavidin (Santa Cruz Biotechnology, CA, USA). Images were obtained by microscope and signal densities were measured and analyzed using the Image-Pro Plus Software (vs.4) program (Media Cybernetics).

\section{Comet assay}

DNA damage was assessed by a single-cell gel electrophoresis assay using a Comet Assay Kit (Trevigen, 4250-050-K) according to the manufacturer's protocol. Briefly, cells were harvested at the indicated times after 6 Gy irradiation, mixed with low-melting-point agarose and plated on the Comet Slide. Cells on the slides were lysed for $30 \mathrm{~min}$ at $4^{\circ} \mathrm{C}$, subjected to electrophoresis at $21 \mathrm{~V}$ for $30 \mathrm{~min}$ under alkaline conditions, and then neutralized and stained with SYBR Green. The presence of comet tails was examined with a Zeiss Axio Observer Z1 fluorescence microscope. Tail moment was calculated as previously described.

\section{Statistical analysis}

Each experiment was repeated at least in triplicate. The data were presented as mean \pm S.E.M and analyzed by SPSS 17.0 software. T-test was used to compare the difference between different groups. $P$ value $<0.05$ was considered significant.

\section{Results}

\section{Twistl confers radioresistance on cervical cancer cells}

To investigate whether Twist1 regulates the radiosensitivity of cervical squamous cancer cells, we constructed lentiviruses for overexpression or knockdown of Twist1 in SiHa cells and performed clonogenic formation assay. Since the viruses constitutively expressed green fluorescent protein (EGFP), the transduction efficiency was determined to be above $90 \%$ based on fluorescent microscopy (Fig. 1A). The cells were named SiHa/Twist1+ (for Twist1 overexpression), $\mathrm{SiHa/Twist1-siRNA-1, \quad \textrm {SiHa } /}$ Twist1-siRNA-2, SiHa/Twist1-siRNA-3 (for Twist1 knockdown), and negative control cells. At least 85\% of Twist1 protein level was knockdown in $\mathrm{SiHa}$ /Twist1-siRNA-2 cells, which showed the strongest inhibition of Twist1 expression in three cell lines infected with Twist1-siRNA-lentivirus (Fig. 1B). The expression of Twist1 in SiHa/Twist1+ cells showed two more times compared with the control (Fig. 1C). The cell lines named Twist1- for 'SiHa/Twist1-siRNA-2' and Twist1+ for 'SiHa/Twist1+' were used in the following experiments. We found that clonogenic survival of Twist1- cells was significantly suppressed upon radiation, while Twist1+ cells exhibited higher resistance to irradiation when compared with negative control cells (Fig. 1D and 1E). These results demonstrate that Twist 1 expression level is negatively correlated with the radiosensitivity of SiHa cells.

\section{Twistl is upregulated in hypoxic cancer cells and promotes radioresistance}

Hypoxia, a solid tumor-specific feature in clinic, is considered as a pivotal factor for tumor radioresistance. Previous study already indicated hypoxia could enhance the Twist1 expression. We then determine whether Twist1 is indeed upregulated in hypoxic tumor cells. We employed hypoxia to treat SiHa cells and detect the express levels of Twist1. Cells were cultured under $1 \%$ oxygen concentrations 
for different time course $(0,4 \mathrm{~h}, 8 \mathrm{~h}, 12 \mathrm{~h}, 24 \mathrm{~h}$ and $48 \mathrm{~h})$ or incubated with hypoxia-mimetic agent $\mathrm{CoCl}_{2}$ $(100 \mathrm{mM})$ for 8 hours. After incubation with hypoxia or hypoxia-mimetic agent $\mathrm{CoCl}_{2}$, HIF-1a expression was induced. Meanwhile, SiHa cells exhibited a significant increase in Twist 1 and the protein level reached the peak at $8 \mathrm{~h}$ after treatment (Fig. 2A and 2B).

To further investigate the role of Twist1 on hypoxia-induced radioresistance, we also examined vector, Twist1- / Twist1+ cells survival by irradiation under hypoxia or normoxia. Cells were incubated under normoxia or hypoxia for $8 \mathrm{~h}$, and then exposed to irradiation. After $72 \mathrm{~h}$, cell clonogenic survival assay was conducted. Indeed, our results indicated that hypoxia increased cell radioresistance, and this effect could be significantly reversed by down-regulation of Twist1 (Fig. 2C). Furthermore, Twist1-depletion did not affect HIF-1a expression (Fig. 2D). Taken together, it is rational that Twist1 may plays a causal role in hypoxia-induced radioresistance in cervical cancer cells.

\section{Twistl enhanced DNA damage repair}

We then addressed the mechanism that Twist1 promotes hypoxia induced radioresistance. After 6Gy radiation treatment, $\gamma \mathrm{H} 2 \mathrm{AX}$ foci was detected by immunofluorescence and western blot. Compared with control SiHa cells, $\gamma \mathrm{H} 2 \mathrm{AX}$ foci persisted much longer in Twist1- cells after irradiation (Fig. 3A and B). Reversely, we hardly observed persistence of $\gamma \mathrm{H} 2 \mathrm{AX}$ foci in Twist1+ cells. Our data indicated that Twist1 expression was contributed to DNA lesions repair. To directly evaluate DNA damage, we performed DNA comet assay to detect double-strand DNA breaks. At $2 \mathrm{~h}$ after ionizing radiation treatment, Twist1- cells exhibited about 2.5-fold increase in the Oliver comet tail moment compared with the control cells (Fig. 3C and D). Oppositely, our data also show that Twist1+ cells decreased comet tail moment by $30 \%$. Collectively, our results demonstrated that Twist1 is required for homologous recombination mediated DNA damage repair and the elimination of DNA breaks.
(A)

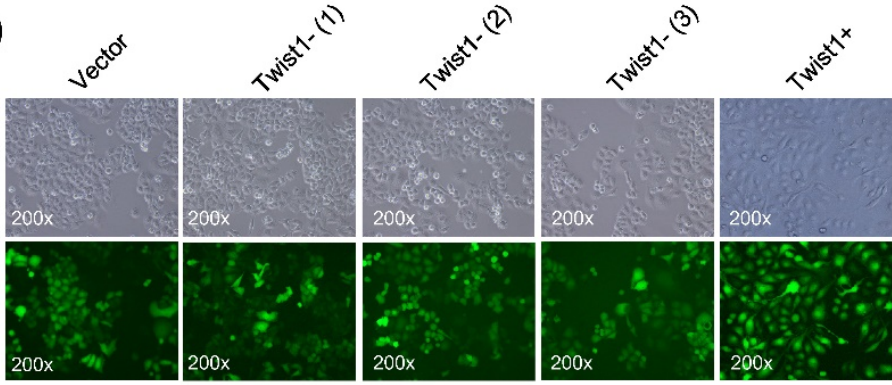

(C)

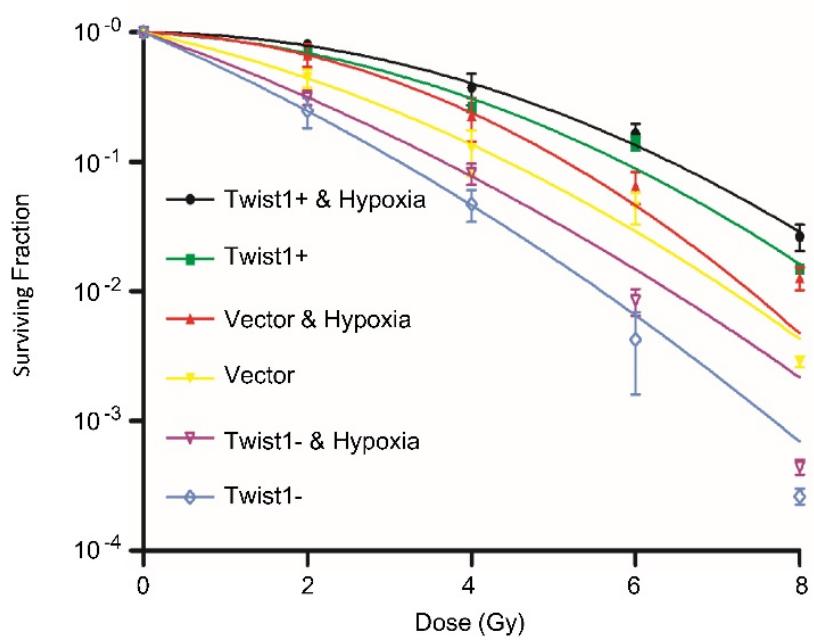

(B)

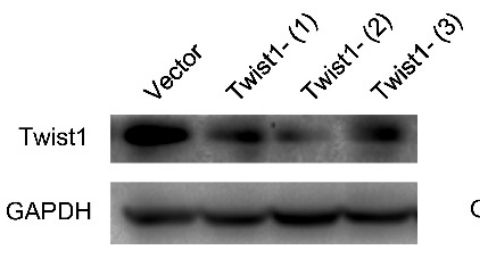

(C)

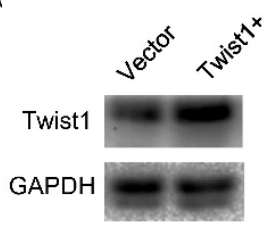

(E)

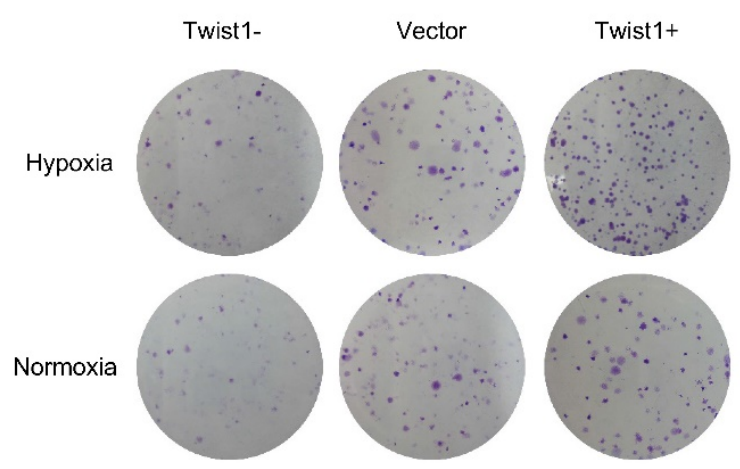

Figure 1. The change of protein levels of Twistl in $\mathrm{SiHa}$ cells after lentivirus infection and following impact on the radiosensitivity of SiHa cells. (A) Fluorescence microscopy of SiHa cells infected with different recombinant lentiviruses. Upper panel: bright field view; lower panel: fluorescence field view (GFP in green). $\times 200$. (B) Protein level of Twistl in negative control cells (Vector) and three different lines of SiHa-Twistl-siRNA cells Twistl- (1), Twist1- (2) and Twistl- (3). (C) Protein level of Twistl in negative control cells (Vector) and SiHa-Twistl+ cells (Twistl+). (D) The survival curve of control cells (Vector), Twistl-overexpression (Twistl+) and Twistl knockdown (Twistl-) SiHa cells under normoxia or hypoxia conditions. (E) Representative photographs of the colony formation assay after irradiation (single dose of 6 Gy). 
(A)

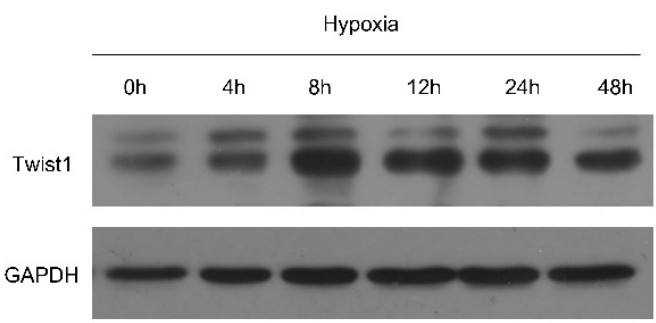

(C)

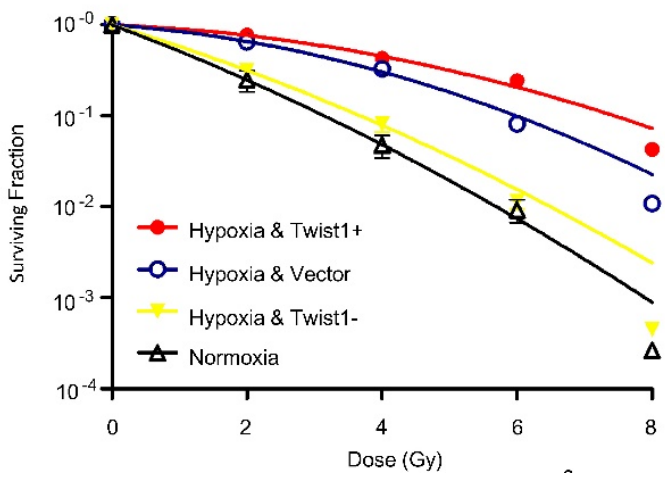

(B)

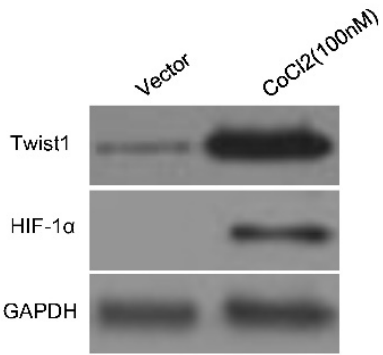

(D)

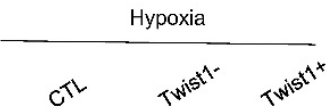

Twist1

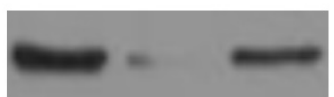

HIF- $1 \alpha$

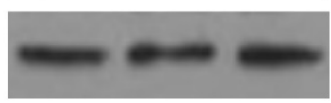

GAPDH

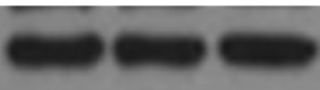

Figure 2. Twistl is up-regultated by hypoxia and promotes hypoxia induced radiorisistance. (A) Protein level of Twistl in SiHa cells cultured under hypoxia for 0 , 12, 24, 48, 72 and $96 \mathrm{~h}$. (B) SiHa cells were treated by $100 \mathrm{mM} \mathrm{CoCl} 2$ for $8 \mathrm{~h}$ and western blot was used to detect Twistl and HIF-1a expression. GAPDH was used as loading control. (C) The survival curve of control cells (Vector). Twistl-overexpression (Twistl+) and Twistl knockdown (Twistl-) SiHa cells under normoxia or hypoxia conditions after irradiation was fitted by linear quadratic (L-Q) model. (D) Twist l + and Twist l- SiHa cells were cultured under hypoxia and the effects of Twistl expression level on HIF-la was detected by western blot.

(A)

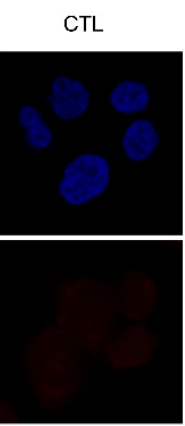

Twist1 +
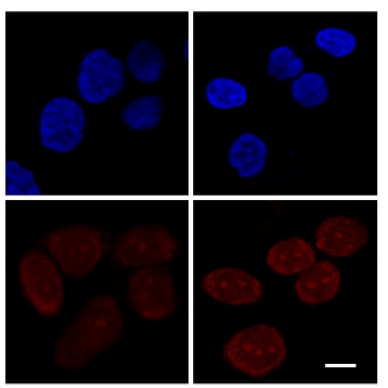

(C)

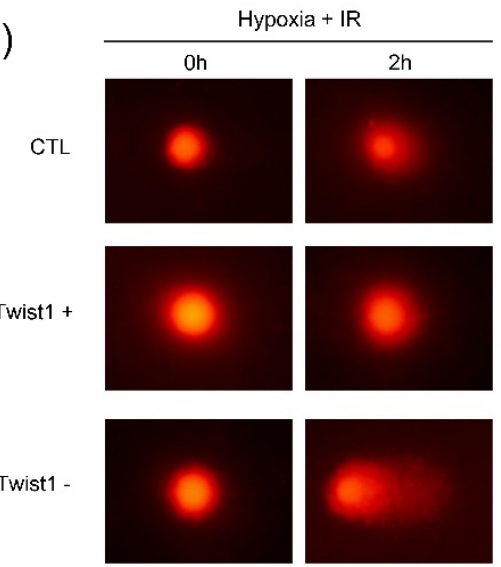

(B)

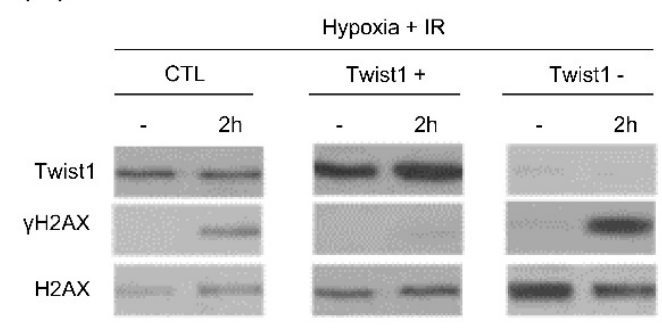

(D)

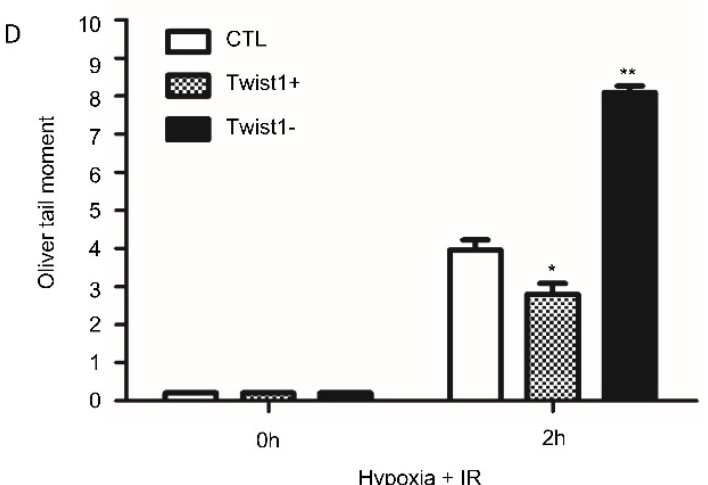

Figure 3. Twist 1 upregulates DNA damage repair (DDR). (A) $2 \mathrm{~h}$ after 6 Gy ionizing radiation, control or Twist $1+/ T$ wist 1 - cells were fixed and H2AX and DAPI staining was performed by immunofluorescence. Scale bar, 20 um. (B) Control or Twist l+/Twistl-cells were cultured under hypoxia and treated with $6 \mathrm{~Gy}$ ionizing radiation. $2 \mathrm{~h}$ after irradiation, cells were lysed and $\mathrm{yH} 2 \mathrm{AX}$ was detected by western blot. (C) and (D) Images (C) and data quantification (D) of singer cell comet assays of control or Twist $1+/$ Twist $1-\mathrm{SiHa}$ cells at the indicated time points after $6 \mathrm{~Gy}$ ionizing radiation. $\mathrm{N}=25$ cells per group. The asterisk $(*)$ indicates a significant decrease $(p<0.05)$ in 16 comet tail moment and the asterisk $(* *)$ indicates a significant $(p<0.01)$ increase. 


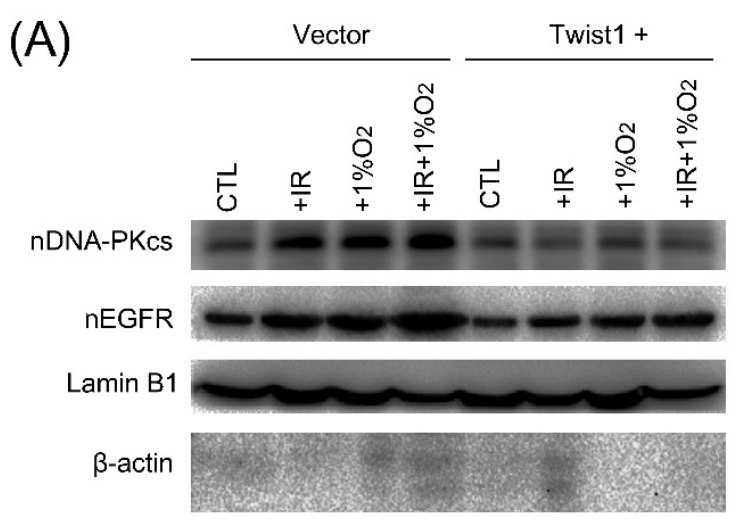

(B)
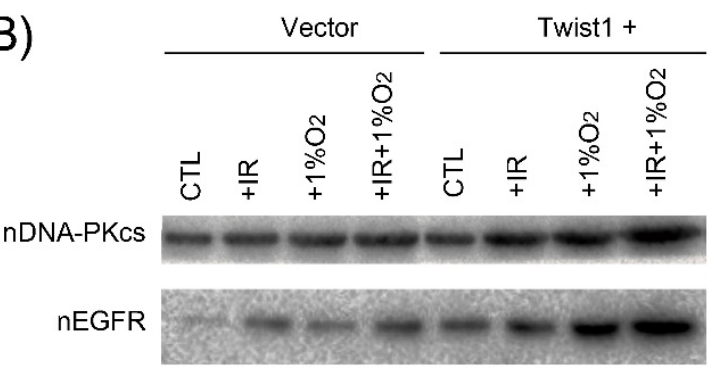

Lamin B1

$\beta$-actin

(C)

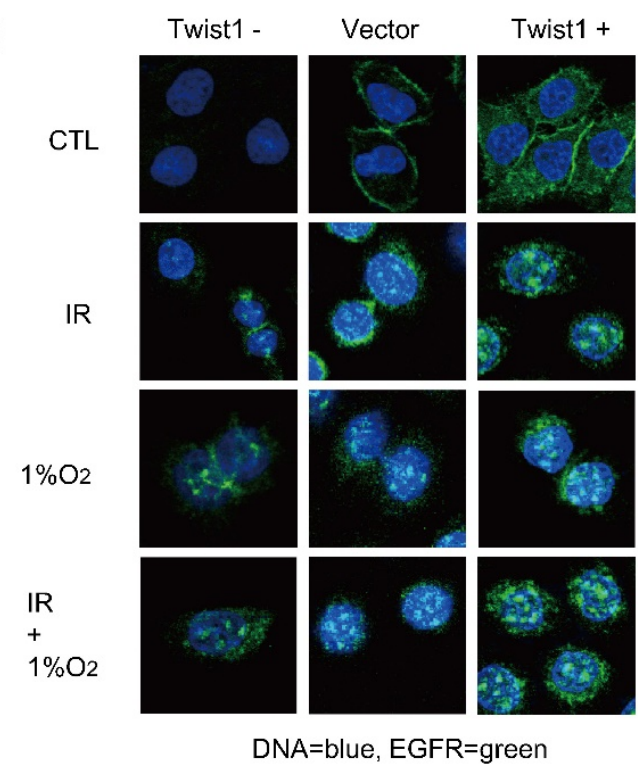

(D)

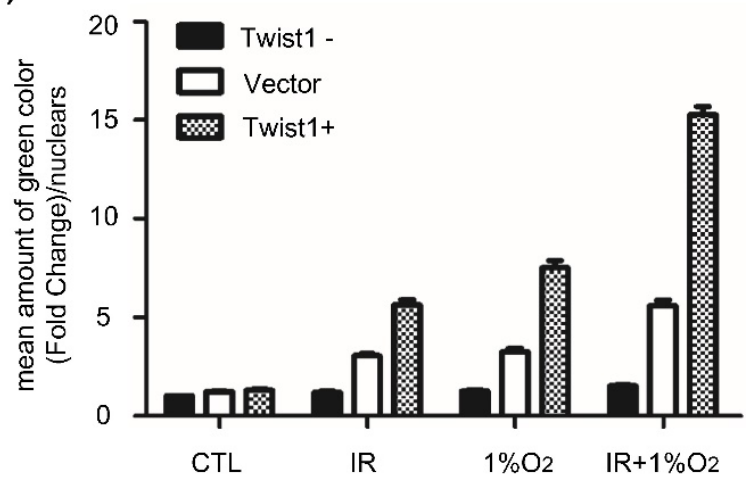

Figure 4. The effects of Twistl expression level on nuclear expression levels of EGFR and DNA-PKcs in SiHa cells. (A) and (B) After treatment with irradiation (5Gy), hypoxia or hypoxia plus irradiation ( $5 \mathrm{~Gy}$ ), the nuclear levels of EGFR and DNA-PKcs in control cells (Vector), Twistl-overexpressing (Twist l+) and Twistl knockdown (Twistl-) SiHa cells were detected by Western blot analysis. Lamin B1 was used as loading control for nuclear proteins. Beta-actin was used to indicate the purity of nuclear supernatants. (C) Representative confocal microscope images of EGFR staining (in green) in control cells (Vector), Twist1-overexpressing (Twist1+) and Twist1 knockdown (Twistl-) SiHa cells untreated (CTL), treated by IR ( $+5 \mathrm{~Gy}$ ) or hypoxia $\left(+\mathrm{CoCl}_{2}\right)$ or both IR and hypoxia $(+5 \mathrm{~Gy} /+\mathrm{CoCl})$. The nuclei were stained by DAPI (in blue). (D) The fluorescence of EGFR staining was quantified with Image J software. For each bar 50 nuclei were evaluated. Data were mean \pm standard error of mean.

\section{Twist 1 regulates nuclear EGFR localization and its downstream DNA-PK activation}

Nuclear EGFR localization and its downstream DNA-PK activation trigger DNA double-strand breaks (DSB) repair. To explore the mechanism underlying Twist1 mediated radioresistance, we examined EGFR in the nuclear fraction and DNA-PKcs. Western blot analysis showed that after exposure to radiation under hypoxia, the amount of nuclear EGFR in Twist1- cells was obviously lower than that in negative control cells. Correspondingly, the expression of nuclear DNA-PKcs was changed along with the nuclear EGFR (Fig. 4A). In contrast, increased levels of nuclear EGFR and DNA-PKcs were detected in Twist1+ cells exposed to radiation (Fig. $4 \mathrm{~B})$. Under normoxia, the same changes of nuclear
EGFR and DNA-PKcs were also observed in Twist1and Twist1+ cells.

Moreover, we investigated the distribution of EGFR upon irradiation under hypoxic stress by confocal microscopy. As shown in Fig. 4C, hypoxia alone increased nuclear EGFR level, and the combination of radiation and hypoxia had minimal additional effect to increase nuclear EGFR level. In addition, hypoxia induced nuclear localization of EGFR was inhibited by Twist1 knockdown but enhanced by Twist1 overexpression (Fig. 4C and D). Collectively, these results demonstrate that Twist1 could increase nuclear EGFR localization and nuclear DNA-PKcs expression, which may mediate the hypoxia-induced radioresistance of cervical cancer cells. 


\section{Twist I correlates with HIF-1 $\alpha$ protein levels and poor clinical outcome in human cervical cancer}

To validate the association between hypoxia and Twist1 expression in cervical cancer patients, we performed tissue-microarray immunohistochemical analysis of HIF-1a and Twist1 expression in 110 sets of human cervical cancer samples. As shown in Fig. 5A, the correlation between Twist1 and HIF-1a was significantly positive $(\mathrm{R}=0.41, p<0.01)$. There were $77.4 \%$ (48 of 62 ) of the tumors with high HIF-1a expression exhibited high Twist1 expression, and $52.1 \%$ (25 of 48 ) of the tumors with low HIF-1a expression showed low Twist1 expression (Fig. 5B).

In clinic, local recurrence after radiotherapy in cervical cancer patients is likely due to tumor radioresistance. To evaluate the clinical prognostic significance of the expression profile of HIF-1a and Twist1, Kaplan-Meier local relapse-free curves were generated and the log-rank test was used to test for significant differences between the groups. The results showed that overexpression of Twist1 correlated with the shorter local relapse-free period (Fig. 5C). Collectively, these data suggest that up-regulation of Twist1 by hypoxia may contribute to

(A)

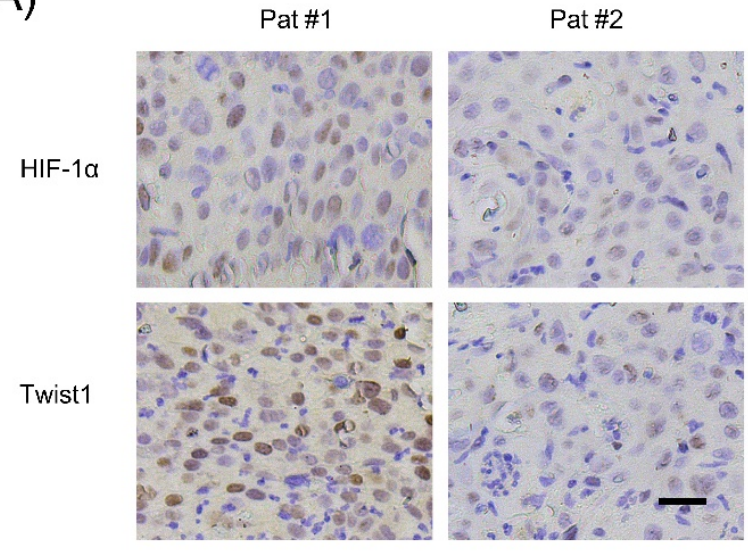

radioresistance and promoting local relapse after irradiation.

\section{Discussion}

Hypoxia has been shown to promote EMT and tumor invasion [17]. Twist1 is a master regulator of EMT and has been implicated in chemoresistance [18]. A recent study showed that silencing Twist1 could enhance IR-induced cell death in NSCLC H1299 and H460 cells [11]. In addition, the analysis of Twist1 expression in cervical cancer patients undergoing post-operative radiotherapy suggested that Twist1 expression level is related to the radiosensitivity and poor prognosis of squamous cell carcinomas, but not in adenocarcinoma [19]. However, the molecular mechanism by which Twist1 regulates radioresistance needs to be elucidated. Given that hypoxia could induce the expression of Twist1 and promote EMT and metastasis of tumor $[9,20,21]$, we hypothesized that Twist1 might be involved in hypoxia-induced radioresistance. Understanding the role of Twist1 in radioresistance particularly in hypoxia condition would benefit the improvement of the efficacy of radiation for cancer treatment.

(B)

\begin{tabular}{|c|c|c|c|}
\hline & HIF-1a-low & HIF-1a-high & Total \\
\hline Twist-low & 25 & 14 & 39 \\
\hline Twist-high & 23 & 48 & 71 \\
\hline Total & 48 & 62 & 110 \\
\hline
\end{tabular}

$p<0.01, R=0.41$

(D)

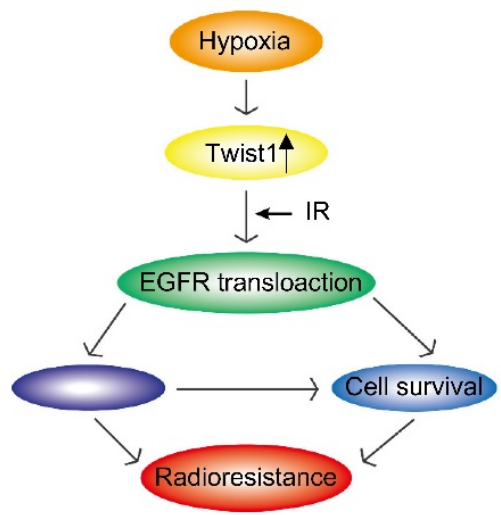

Figure 5. Twistl correlates with HIF-1 $\alpha$ protein levels and poor clinical outcome in human cervical cancer. (A) Immunohistochemical staining of Twistl and HIF-1 1 in representative cancer specimens on cervical cancer tissue microarrays. Brown staining indicates positive immunoreactivity. Scale bar, 25um. (B) Correlation between Twistl and HIF-1 a protein levels in human cervical tumors. Statistical significance was determined by $\chi^{2}$ test. $R$ is the correlation coefficient. (C) Kaplan-Meier curves show the local relapse-free survival of patients with high or low expression of Twistl in their cervical tumors. Statistical significance was determined by a log-rank test. (D) The mechanic model of regulation of radiosensitivity and DDR by Twist 1 . 
The present study utilized SiHa cervical carcinoma cell line, which was described as a squamous carcinoma cell line [22] and exhibited medium level of Twist1 expression among four cervical cell lines (data not shown). Previous study indicated that HIF-1a could bind directly to the hypoxia-response element (HRE) in Twist1 promoter to enhance Twist1 expression [9]. We observed that HIF-1a expression was induced under hypoxia condition in SiHa cells and the expression of Twist1 was also increased in $\mathrm{SiHa}$ cells exposed to hypoxia. The phenomena that hypoxia could upregulate the expression of Twist1 suggest that it would be meaningful to explore the role of Twist1 in radiation sensitivity in hypoxic cells. By manipulating Twist1 expression, we observed that knockdown of Twist1 could significantly facilitate the radiosensitivity of $\mathrm{SiHa}$ cells both in normoxia and hypoxia conditions. On the contrary, overexpression of Twist1 could reduce the radiosensitivity of $\mathrm{SiHa}$ cells. To the best of our knowledge, this was the first study to explore the role of Twist1 in radioresistance in hypoxia condition. Additionally, we found that $\mathrm{SiHa}$ cells upon ionizing irradiation exhibited a minimal increase in Twist1 expression and the peak level was at $24 \mathrm{~h}$ after IR with 5 Gy (Data not shown), very similar to previous report in endometrial carcinoma HEC1A cells [23].

Seo et al. reported that knockdown of Twist1 could promoted IR-induced apoptosis in NSCLC cells [11], which may explain how Twist1 contributes to radioresistance in cancer cells. DNA repair pathway which involves IR-induced EGFR up-regulation and subsequent activation of DNA-PKcs is another important mechanism to regulate radiosensitivity [24]. Emerging evidence demonstrated that nuclear EGFR not only contributed to cancer progression, but also enhanced chemo- and radio-resistance [12, 25, 26]. Upon shuttling into the nucleus, nuclear EGFR can function as a co-transcription factor for a series of oncogenes and phosphorylate proliferating cell nuclear antigen (PCNA) to accelerate cellular proliferation [27-29]. Notably, nuclear localized EGFR, forming a complex with DNA-PK, triggers DNA repair and results in radiation resistance [30]. Therefore, we investigated whether the interference of Twist1 expression could affect nuclear EGFR localization and associated DNA-PK expression in $\mathrm{SiHa}$ cells under normoxia and hypoxia condition. We observed that silencing Twist1 decreased nuclear localization of EGFR and DNA-PKcs level in irradiated cells compared to control cells, while nuclear localization of EGFR and DNA-PKcs level increased in Twist1-overexpressing SiHa cells. Confocal microscopy further confirmed that Twist1 knockdown significantly inhibited hypoxia induced nuclear localization of EGFR while Twist1 overexpression significantly promoted hypoxia induced nuclear localization of EGFR. Taken together, these results suggest that Twist1 is responsible for the nuclear translocation of EGFR under hypoxia condition, and increased nuclear levels of EGFR then mediated the radioresistance of cervical cancer cells. However, the mechanism by which Twist1 modulates the nuclear localization of EGFR needs to be further studied.

In conclusion, for the first time we report the close associations between hypoxia and Twist1 level, nuclear location of EGFR and nuclear level of DNA-PKcs in cancer cells. Hypoxia upregulates Twist1 expression, which in turn contributes to hypoxia-induced radioresistance. Knockdown of Twist1 remarkably enhances radiosensitivity of cancer cells, accompanied by diminished nuclear stabilization of EGFR and decreased nuclear levels of DNA-PK. Our data provide new insights into molecular mechanism underlying hypoxia-induced radioresistance in cervical cancer cells, and suggest that Twist1 is a promising molecular target to reverse cancer radioresistance especially in hypoxia condition.

\section{Abbreviations}

EGFR: epidermal growth factor receptor; HIF-1: Hypoxia-inducible factor 1; siRNA: short interfering RNA; EMT: epithelial-mesenchymal transition; NSCLC: non-small-cell lung cancer; DNA-PK: DNA-dependent protein kinase; EGFP: expressed green fluorescent protein; DSB: DNA double-strand breaks; HRE: hypoxia-response element; PCNA: proliferating cell nuclear antigen; DDR: DNA damage repair.

\section{Acknowledgments}

This work was supported by grant No. 81372434 (Huihua Xiong) from the National Natural Science Foundation; Grant No. 81502118 (Yanmei Zou) from the National Natural Science Youth Foundation.

\section{Competing Interests}

The authors have declared that no competing interest exists.

\section{References}

1. Jemal A, Bray F, Center MM, Ferlay J, Ward E and Forman D. Global cancer statistics. CA Cancer J Clin. 2011; 61(2):69-90.

2. Qin $C$, Chen $X$, Bai $Q$, Davis $M R$ and Fang $Y$. Factors associated with radiosensitivity of cervical cancer. Anticancer Res. 2014; 34(9):4649-4656.

3. Vaupel P and Mayer A. Hypoxia in cancer: significance and impact on clinical outcome. Cancer Metastasis Rev. 2007; 26(2):225-239.

4. Huang M, Chen Q, Xiao J, Yao T, Bian L, Liu C and Lin Z. Overexpression of hypoxia-inducible factor-1alpha is a predictor of poor prognosis in cervical cancer: a clinicopathologic study and a meta-analysis. Int J Gynecol Cancer. 2014; 24(6):1054-1064. 
5. Yang $Y$, Yang $X$, Zhu H, Guo Q, Chen X, Zhang H, Cheng $H$ and Sun $X$. Autophagy and its function in radiosensitivity. Tumour Biol. 2015; 36(6):4079-4087.

6. Moeller BJ, Richardson RA and Dewhirst MW. Hypoxia and radiotherapy: opportunities for improved outcomes in cancer treatment. Cancer Metastasis Rev. 2007; 26(2):241-248.

7. Huang Y, Yu J, Yan C, Hou J, Pu J, Zhang G, Fu Z and Wang X. Effect of small interfering RNA targeting hypoxia-inducible factor-1alpha on radiosensitivity of PC3 cell line. Urology. 2012; 79(3):744 e717-724.

8. Jung HY and Yang J. Unraveling the TWIST between EMT and cancer stemness. Cell Stem Cell. 2015; 16(1):1-2.

9. Yang MH, Wu MZ, Chiou SH, Chen PM, Chang SY, Liu CJ, Teng SC and Wu KJ. Direct regulation of TWIST by HIF-1alpha promotes metastasis. Nat Cell Biol. 2008; 10(3):295-305.

10. Sun S, Ning X, Zhang Y, Lu Y, Nie Y, Han S, Liu L, Du R, Xia L, He L and Fan D. Hypoxia-inducible factor-1alpha induces Twist expression in tubular epithelial cells subjected to hypoxia, leading to epithelial-to-mesenchymal transition. Kidney Int. 2009; 75(12):1278-1287.

11. Seo SK, Kim JH, Choi HN, Choe TB, Hong SI, Yi JY, Hwang SG, Lee HG, Lee $\mathrm{YH}$ and Park IC. Knockdown of TWIST1 enhances arsenic trioxide- and ionizing radiation-induced cell death in lung cancer cells by promoting mitochondrial dysfunction. Biochem Biophys Res Commun. 2014; 449(4):490-495.

12. Brand TM, Iida M, Luthar N, Starr MM, Huppert EJ and Wheeler DL. Nuclear EGFR as a molecular target in cancer. Radiother Oncol. 2013; 108(3):370-377.

13. Dittmann K, Mayer C, Fehrenbacher B, Schaller M, Kehlbach R and Rodemann HP. Nuclear epidermal growth factor receptor modulates cellular radio-sensitivity by regulation of chromatin access. Radiother Oncol. 2011; 99(3):317-322.

14. Liccardi G, Hartley JA and Hochhauser D. EGFR nuclear translocation modulates DNA repair following cisplatin and ionizing radiation treatment. Cancer Res. 2011; 71(3):1103-1114.

15. Jette $\mathrm{N}$ and Lees-Miller SP. The DNA-dependent protein kinase: A multifunctional protein kinase with roles in DNA double strand break repair and mitosis. Prog Biophys Mol Biol. 2015; 117(2-3):194-205.

16. Bae S, Jeong HJ, Cha HJ, Kim K, Choi YM, An IS, Koh HJ, Lim DJ, Lee SJ and An S. The hypoxia-mimetic agent cobalt chloride induces cell cycle arrest and alters gene expression in U266 multiple myeloma cells. Int J Mol Med. 2012; 30(5):1180-1186

17. Lei J, Fan L, Wei G, Chen X, Duan W, Xu Q, Sheng W, Wang K and Li X. Gli-1 is crucial for hypoxia-induced epithelial-mesenchymal transition and invasion of breast cancer. Tumour Biol. 2015; 36(4):3119-3126.

18. Lu S, Yu L, Mu Y, Ma J, Tian J, Xu W and Wang H. Role and mechanism of Twist1 in modulating the chemosensitivity of FaDu cells. Mol Med Rep. 2014; 10(1):53-60.

19. Shibata K, Kajiyama H, Ino K, Terauchi M, Yamamoto E, Nawa A, Nomura S and Kikkawa F. Twist expression in patients with cervical cancer is associated with poor disease outcome. Ann Oncol. 2008; 19(1):81-85.

20. Luo $\mathrm{D}$, Wang $\mathrm{Z}, \mathrm{Wu}$ J and Jiang $\mathrm{C}$. The role of hypoxia inducible factor- 1 in hepatocellular carcinoma. Biomed Res Int. 2014; 2014:409272.

21. Yang MH and Wu KJ. TWIST activation by hypoxia inducible factor-1 (HIF-1): implications in metastasis and development. Cell Cycle. 2008; 7(14):2090-2096.

22. Kurban S, Tursun M, Kurban G and Hasim A. Role of CXCR7 and effects on CXCL12 in SiHa cells and upregulation in cervical squamous cell carcinomas in Uighur women. Asian Pac J Cancer Prev. 2014; 15(21):9211-9216.

23. Tsukamoto H, Shibata K, Kajiyama H, Terauchi M, Nawa A and Kikkawa F. Irradiation-induced epithelial-mesenchymal transition (EMT) related to invasive potential in endometrial carcinoma cells. Gynecol Oncol. 2007; 107(3):500-504.

24. Zhou X, Zhang X, Xie Y, Tanaka K, Wang B and Zhang H. DNA-PKcs inhibition sensitizes cancer cells to carbon-ion irradiation via telomere capping disruption. PLoS One. 2013; 8(8):e72641.

25. Li C, Iida M, Dunn EF, Ghia AJ and Wheeler DL. Nuclear EGFR contributes to acquired resistance to cetuximab. Oncogene. 2009; 28(43):3801-3813.

26. Huang WC, Chen YJ, Li LY, Wei YL, Hsu SC, Tsai SL, Chiu PC, Huang WP, Wang $\mathrm{YN}$, Chen $\mathrm{CH}$, Chang WC, Chen AJ, Tsai $\mathrm{CH}$ and Hung MC. Nuclear translocation of epidermal growth factor receptor by Akt-dependent phosphorylation enhances breast cancer-resistant protein expression in gefitinib-resistant cells. J Biol Chem. 2011; 286(23):20558-20568.

27. Lo HW, Cao X, Zhu H and Ali-Osman F. Cyclooxygenase-2 is a novel transcriptional target of the nuclear EGFR-STAT3 and EGFRvIII-STAT3 signaling axes. Mol Cancer Res. 2010; 8(2):232-245.

28. Yu YL, Chou RH, Wu CH, Wang YN, Chang WJ, Tseng YJ, Chang WC, Lai CC, Lee HJ, Huo L, Chen $\mathrm{CH}$ and Hung MC. Nuclear EGFR suppresses ribonuclease activity of polynucleotide phosphorylase through DNAPK-mediated phosphorylation at serine 776. J Biol Chem. 2012; 287(37):31015-31026.

29. Wang SC, Nakajima Y, Yu YL, Xia W, Chen CT, Yang CC, McIntush EW, Li LY, Hawke DH, Kobayashi R and Hung MC. Tyrosine phosphorylation controls PCNA function through protein stability. Nat Cell Biol. 2006; 8(12):1359-1368

30. Dittmann K, Mayer C, Kehlbach R and Rodemann HP. Radiation-induced caveolin-1 associated EGFR internalization is linked with nuclear EGFR transport and activation of DNA-PK. Mol Cancer. 2008; 7:69. 